\title{
Ultrathin Films of YBaCuO Grown on YSZ Substrates with a New Buffer Layer Nd-Cu-O
}

\author{
J. Gao, T.C. Chui, and W.H. Tang \\ Department of Physics, the University of Hong Kong, Pokfulam Road, Hong Kong
}

\begin{abstract}
YSZ is one of the most widely used substrates to grow oxide superconducting thin films. However, the large lattice mismatch and occurrence of intermediate layer often degrade the quality of grown films. We used a new buffer material, neodymium copper oxide $\left(\mathrm{Nd}_{2} \mathrm{CuO}_{4}\right)$, to improve the quality of YBCO ultrathin films grown on YSZ. Good superconducting transitions have been obtained on these films with thickness 10 12 nanometers. Such a $\mathrm{Nd}_{2} \mathrm{CuO}_{4}$ layer has a stable crystal structure and behaves as an oxide semiconductor. At $77 \mathrm{~K}$, its resistivity is $\sim \mathbf{4 0}$ times higher than that of $\mathrm{PrBaCuO}$, which has been commonly used as buffer layer and barrier material. The lattice mismatch between $\mathrm{Nd}_{2} \mathrm{CuO}_{4}$ and $\mathrm{YBCO}$ is much smaller than that between YSZ and YBCO. It allows highly epitaxial growth of YBCO without forming big defects and the intermediate layer. Measurements by using XRD, rocking curve, and cross-sectional TEM indicated that the crystallinity of YBCO films grown on $\mathrm{Nd}_{2} \mathrm{CuO}_{4}$ buffer layer had been significantly improved. Besides, the smooth surface of $\mathrm{Nd}_{2} \mathrm{CuO}_{4}$ layer and buffered YBCO films are observed by SEM.
\end{abstract}

\section{INTRODUCTION}

Yttrium stabilized $\mathrm{ZrO}_{2}$ (YSZ) is one of the most commonly used substrates to grow oxide superconducting thin films. However, the large lattice mismatching and occurrence of intermediate layer often degrade the quality of grown films [1]. The problems become more serious in the preparation of ultrathin films with a thickness of only a few unit cells. To improve the lattice matching and reduce the interaction between $\mathrm{YBa}_{2} \mathrm{Cu}_{3} \mathrm{O}_{\mathrm{y}}(\mathrm{YBCO})$ and $\mathrm{YSZ}$, we have utilized a new buffer material $\mathrm{Nd}_{2} \mathrm{CuO}_{4}(\mathrm{NCO})$ as buffer layer on YSZ substrates. High quality $\mathrm{YBCO}$ thin and ultrathin films with improved crystallinity and good superconducting properties have been successfully fabricated. $\mathrm{NCO}$ material, behavior as a semiconductor, was first reported in 1973 [2]. Such a NCO material has a stable tetragonal structure with lattice constants $a=3.86 \AA$ and $c=$ $12.50 \AA$. It behaves as a semiconductor and possesses a high resistivity. In particular the NCO layer can be grown under the deposition conditions similar to that for making YBCO thin films. Noteworthy, NCO is a parental material of the hole-carrier and electron-carrier superconductors, such as $\mathrm{Nd}_{2} \mathrm{CuO}_{4-\mathrm{x}} \mathrm{F}_{\mathrm{x}}$ and $\mathrm{Nd}_{2-\mathrm{x}} \mathrm{Ce}_{\mathrm{x}} \mathrm{CuO}_{4}$, although NCO itself is nonsuperconductive [3], [4]. The nature of NCO buffer layer, growth of $\mathrm{NCO}$ and $\mathrm{YBCO}$ films, and their structural and electric properties will be discussed in this paper.

Manuscript received September 14, 1998

This research project has been supported by the Hong Kong Research Grants Council (RGC).

\section{EXPERIMENT}

All YBCO thin films and NCO buffer layers were fabricated by using an off-axis rf-magnetron sputter technique [5], [6]. (100) YSZ single crystal has been used as substrate. Sintered stoichiometric YBCO ceramics disc with a diameter of 50 $\mathrm{mm}$ and thickness $\sim 4 \mathrm{~mm}$ was used as sputter target. The chamber was evacuated to pressure less than $5 \times 10^{-6}$ mbar before the sputtering. A mixture of argon and oxygen with a ratio of $\mathrm{Ar} / \mathrm{O}_{2}-3: 1$ was used as sputter gas. During deposition process, the total sputter pressure was kept at 0.1 0.2 mbar. Rf-power of $70 \mathrm{~W}$ was applied on the cathode, generating a power density $\sim 3.6 \mathrm{~W} / \mathrm{cm}^{2}$. The substrate temperature for growing $\mathrm{NCO}$ and $\mathrm{YBCO}$ were $780^{\circ} \mathrm{C}$ and $750{ }^{\circ} \mathrm{C}$, respectively. These substrate temperatures were determined by a k-type thermocouple inserted into the heater.

NCO-buffered YBCO thin and ultrathin films were characterized by using $\mathrm{X}$-ray diffraction, transmission electron microscope, scanning electron microscopy, standard fourprobe measurement etc. The thickness of the film was determined by a Dektak3ST surface step profiler. The average height of the step is defined as the film thickness. To facilitate the surface scan of profiler accurately, the well-defined and very sharp steps of micro-bridge were patterned by using photolithography technique.

\section{RESULT AND DISCUSSION}

Under the deposition conditions described above, both NCO buffer layer and YBCO film are grown with the $c$-axis orientation. The typical X-ray spectrum of our YBCO films

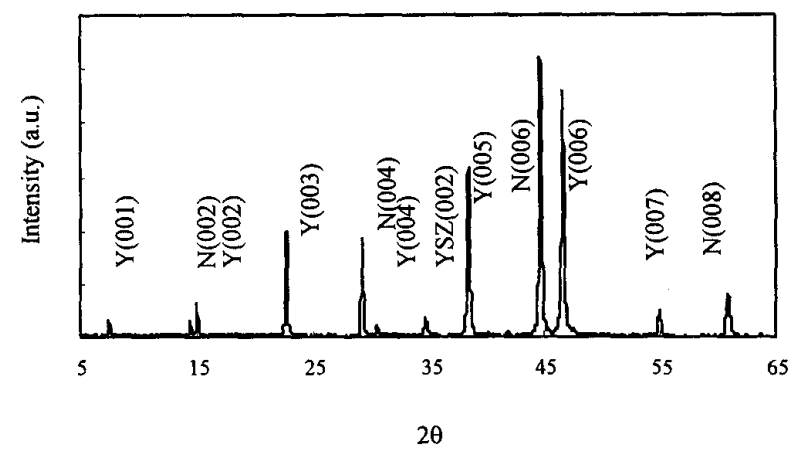

Fig. 1 The $\mathrm{x}$-ray spectrum of $(00 l)$-oriented $\mathrm{YBCO}$ thin films on YSZ substrate with $(002 I)$-oriented $\mathrm{NCO}$ buffer layer. 
grown on (002l)-oriented NCO buffer layer is shown in Fig. 1. In this figure, the peaks are labeled and divided into three groups, namely YBCO (Y), NCO (N), YSZ. The coherent orientations of $\mathrm{YBCO}$ and $\mathrm{NCO}$ are obtained in the spectrum. Besides the reflection from substrate and the $(00 l)$ peaks of $\mathrm{YBCO}$ and $\mathrm{NCO}$, no other peaks are visible, demonstrating that the films are absent of other phases. During the deposition of $\mathrm{NCO}$, the substrate temperature was slightly increased to $780^{\circ} \mathrm{C}$ since a higher temperature can significantly suppress the undesirable (103)-orientation of NCO. The full width half maximum (FWHM) of the rocking curves of the (005) peak of YBCO was measured $<0.35^{\circ}$, indicating a highly epitaxy and good crystal structure of the grown films with the NCO buffer layer.

For a buffer material a smooth film surface is a prerequisite to further grow YBCO thin and ultrathin films. A good surface morphology is a very desirable property for choosing $\mathrm{NCO}$ as the buffer layer. A typical SEM image of a NCO buffer layer on YSZ with the magnification of $20 \mathrm{~K}$ is shown in Fig. 2a. The layer is quite flat and smooth over a large area. No big surface structures can be found except some small droplets with a typical size $\sim 0.3 \mu \mathrm{m}$. These droplets could be identified as the copper-rich phase of NCO material by Energy Dispersive X-ray Analysis (EDAX). Investigated by using a surface step profiler the average surface roughness is found being about a few nanometers. The thickness of this $\mathrm{NCO}$ film is $1.05 \mathrm{~nm}$. Usually with such a value of film thickness the surface of YBCO or other materials like $\mathrm{PrBa}_{2} \mathrm{Cu}_{3} \mathrm{O}_{\mathbf{x}}$ will become much rough and many outgrowths could be formed.

Usually the surface morphology of the film grown on a buffered substrate is poor in comparing with those directly grown on bare substrates. However, our YBCO thin films made on the NCO buffered YSZ still show a very smooth surface. Fig. $2 \mathrm{~b}$ presents a typical SEM image of a $90 \mathrm{~nm}$ YBCO film grown on YSZ substrate with $10 \mathrm{~nm}$ NCO buffer layer. By comparing this figure to Fig. 2a, they have almost same smoothness except $\sim 0.3 \mu \mathrm{m}$ sized outgrowths increase a little. Noteworthy, some small outgrowths, with size $\sim 50 \mathrm{~nm}$, are found in same photo. The film surface quality does not

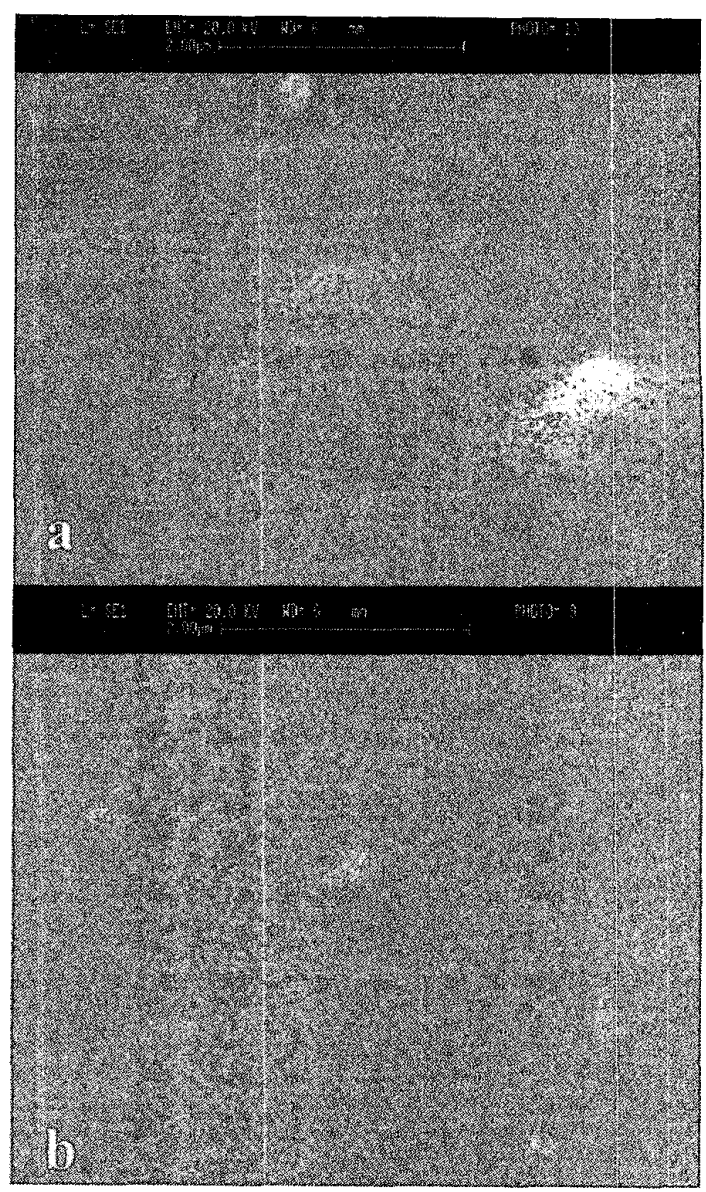

Fig.2. The SEM photos of (a) $105 \mathrm{~nm}$ NCO layer (b) $90 \mathrm{~nm}$ YBCO films with $10 \mathrm{~nm}$ NCO buffer layer

significantly differ from that of our YBCO thin and ultrathin films grown on bare YSZ [7].

To explore the quality of epitaxy and the microstructure in these films, cross sectional transmission electron microscope

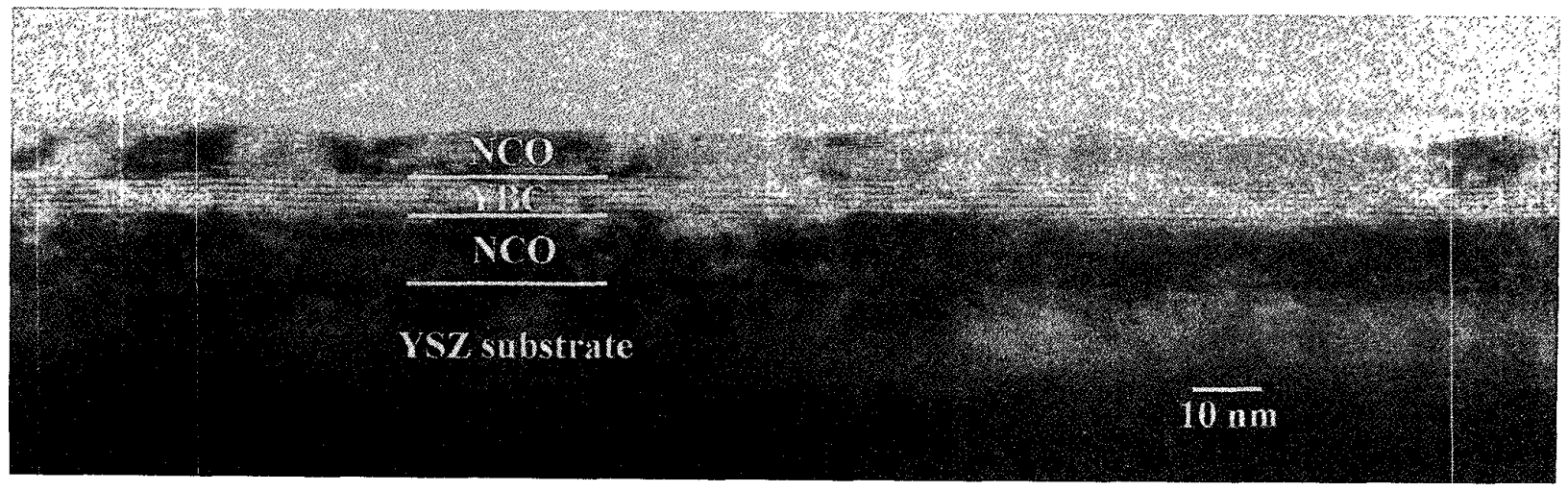

Fig.3. The cross-sectional TEM photo of a NCO sandwiched YBCO ultrathin film with a film thickness about 5-unit cells 
(XTEM) study was carried out. The details of preparation of XTEM samples have been described earlier [8]. Fig. 3 shows an image of a 5-unit-cell YBCO ultrathin film sandwiched by NCO films on a YSZ substrate. A thin layer of NCO was grown onto the $\mathrm{YBCO}$ to protect the sample from surface damage by the mechanical sample processing for the crosssectional TEM studies. Our ultrathin film sample were milled at room temperature. As can be seen in the XTEM picture, the YBCO ultrathin film grown on the NCO buffer layer is highly epitaxially. The film thickness of YBCO is only $6 \mathrm{~nm}$, about 5 unit cells. The NCO buffer and protect layers are about $10 \mathrm{~nm}$ and $6 \mathrm{~nm}$, respectively. Three layers are marked separately. The brightest dots correspond to the $\mathrm{Cu}$ between $\mathrm{BaO}$ planes. The parallel lines of the spots are along $a$-direction of YBCO. From the image we estimate that the spatial differences between two bright lines are $\sim 1.2 \mathrm{~nm}$, i.e. the c-lattice constant of YBCO. The continuous CuO planes with length longer that $200 \mathrm{~nm}$ observed by TEM indicate the superior crystal structure of the buffered ultrathin film sample. Even at this very early growth stage, our YBCO film has already formed a continuous layer. It underlines the excellent growth of our YBCO ultrathin films on NCO buffered YSZ.

In contrast, the ultrathin films grown on YSZ without a buffer showed relatively poor crystallinity. Various defects, like secondary phases and Ba-depletion of YBCO, could be formed due to the chemical formation of the intermediate layer material $\left(\mathrm{BaZrO}_{3}\right)$ [1]. In our case, the chemical interaction between the YBCO film and YSZ has been buffered by the NCO layer. A clear interface between NCO and YSZ can be seen in the TEM image. NCO is also chemically compatible with YBCO. The interface between YBCO and NCO is sharp and hardly any interaction could be observed. Although a trifling interdiffusion might take place at the interface, mainly the $\mathrm{Nd}^{3+}$ replaces $\mathrm{Y}^{3+}$, it would not result in any detrimental effect. Obviously, the use of the NCO buffer layer has significantly improved the growth of the YBCO layer.

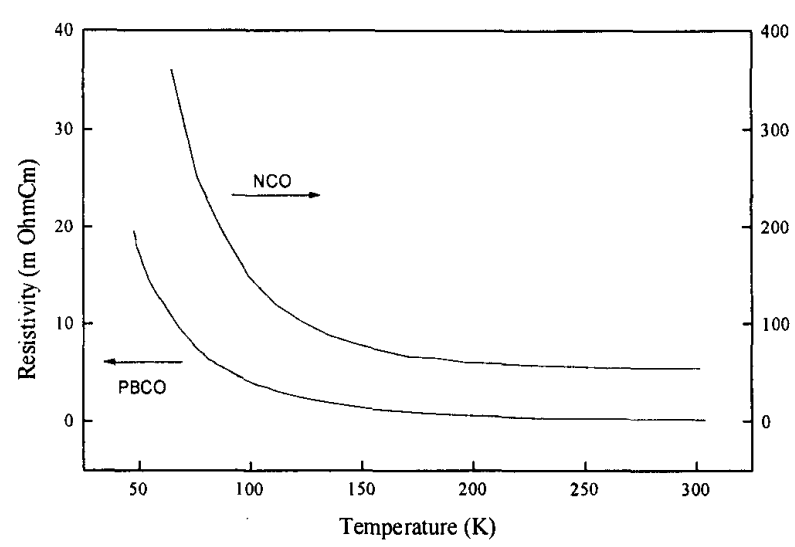

Fig. 4. The temperature dependence of resistivity of NCO and $\mathrm{PBCO}$ thin films
The NCO material is known as a semiconductor, similar to $\mathrm{PrBa}_{2} \mathrm{Cu}_{3} \mathrm{O}_{\mathrm{x}}$. The temperature dependence of its resistivity is compared with that of a $\mathrm{PrBa}_{2} \mathrm{Cu}_{3} \mathrm{O}_{\mathrm{x}}$ film in Fig. 4. At the boiling point of liquid nitrogen the resistivity of $\mathrm{NCO}$ and PBCO are 250 and $7 \mathrm{~m} \Omega \cdot \mathrm{cm}$, respectively. In other words, the resistivity of $\mathrm{NCO}$ is $\sim 40$ times larger than that of $\mathrm{PBCO}$ thin films. Moreover, the increasing rate of resistivity with temperature of $\mathrm{NCO}$ is much faster than that of $\mathrm{PrBa}_{2} \mathrm{Cu}_{3} \mathrm{O}_{\mathrm{x}}$. Even at room temperature the resistivity of $\mathrm{NCO}$ layer is about 700 times higher than that of YBCO. Thus the influence of the NCO buffer layer is negligible in most electric measurements. The very high resistivity of NCO makes it superior to $\mathrm{PBCO}$ for the applications as a buffer layer or a barrier material. Since the resistivity of these oxide materials can be affected by oxygen contents, both materials are deposited under similar conditions for this comparison.

The direct effect of inserting a buffer layer between YBCO and YSZ is to block their interaction and reduce the lattice mismatching. A question raised is what is the minimum thickness of the NCO layer needed to efficiently block the interaction? From our experiments we found that a $10 \mathrm{~nm}$ NCO layer can sufficiently buffer the interaction between YBCO and YSZ. As revealed by the XTEM image shown in Fig. 3, no intermediate layer was formed at the substrate interface. The structural strain caused by the lattice mismatching was studied by electron backscatter diffraction (EBSD). It was found that the strain was remarkably reduced in the buffered YBCO films, even with a very thin NCO intermediate layer. As the deposition rate of the NCO material is much lower than that of YBCO, for most of our samples we used a $10 \mathrm{~nm}$ NCO layer as the buffer.



Fig. 5. The critical temperature and normal-state resistivity of YBCO grown on a $10 \mathrm{~nm}-\mathrm{NCO}$ buffer layer are plotted as a function of the thickness of the YBCO layer. 


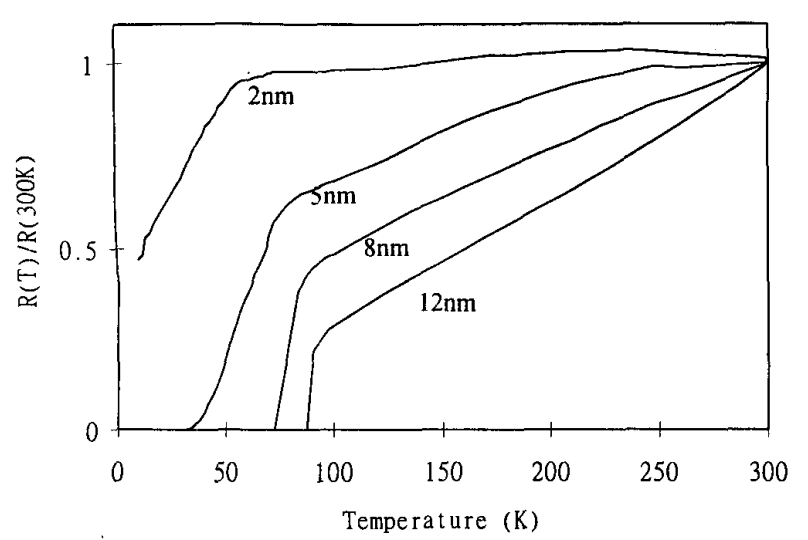

Fig. 6. Typical transition curves for the different thickness of YBCO thin films on fixed $10 \mathrm{~nm}-\mathrm{NCO}$ buffer layer.

Our aim in this work is to improve the growth of YBCO ultra thin films by using such a new buffer layer. The influence of the film thickness of YBCO on their superconducting properties has been systemically studied. In Fig. 5 the transition temperature and the ratio of the resistance at $300 \mathrm{~K}$ and $100 \mathrm{~K}(R R R)$ is plotted against the YBCO layer thickness. All samples presented in this figure were deposited on a $10 \mathrm{~nm} \mathrm{NCO}$ buffer layer. It can be seen that there is no significant degradation of superconductivity for YBCO films down to $12 \mathrm{~nm}$. The films showed a strong metallic behavior. The typical superconducting transition curves of these YBCO ultrathin films are given in Fig. 6. By using a NCO buffer, full superconducting transitions with $T_{\mathrm{c}, \text { zero }} \geq 87 \mathrm{~K}$ have be obtained on $12 \mathrm{~nm}$ YBCO ultrathin film. When the film thickness of YBCO decreases to $8 \mathrm{~nm}$ the $T_{\mathrm{c}, \text { zero }}$ is degraded to $70 \mathrm{~K}$ and $R R R$ decreases from 3 to 2.4. No complete superconducting transition could be found for ultrathin films of 1-2 nm although the $T_{\text {onset }}$ was still above $50 \mathrm{~K}$. The results are similar to that reported in YBCO ultrathin films grown on YSZ bare substrates and grown on other buffer material $\mathrm{La}_{2} \mathrm{CuO}_{4}[9],[10]$. But the crystallinity and epitaxy of our samples are significantly improved as revealed by rocking curve measurements, EBSD, and TEM studies.

\section{CONCLUSION}

$(00 l)$-oriented YBCO thin and ultrathin films are grown on the YSZ substrates with $(002 l)$-oriented NCO thin buffer layers. Such a NCO buffer layer possesses a stable structure, which does not react with the YSZ form a $\mathrm{BaZrO}_{3}$ intermediate layer, and results in a very smooth surface. In addition, its close lattice matching with YBCO and high resistivity make the $\mathrm{NCO}$ material promising for applications like buffer and barrier materials. It has been found that a 10 $\mathrm{nm}$ NCO layer is sufficient to buffer the interaction between YBCO and YSZ substrate. The crystallinity and epitaxy of the buffered YBCO is significantly improved and good superconducting properties, such as $T_{\mathrm{c}}$ and $J_{\mathrm{c}}(77 \mathrm{~K})$, remain.

\section{ACKNOWLEDGMENT}

The authors would like to thank Ms Y. Yang for carrying out the TEM study. The SEM and EBSD measurements were made in the Microscope Unit of the University of Hong Kong.

\section{REFERENCES}

[1] J. G. Wen, C. Traeholt, H. W. Zandbergen, K. Joosse, E. M. C. M. Reuvekamp, and H. Rogalla, "A HRTEM study of the atomic structure and the growth mechanism at the $\mathrm{YBa}_{2} \mathrm{Cu}_{3} \mathrm{O}_{7} / \mathrm{YSZ}$ interface,"Physica $C$, vol. 218, pp. 29-42, 1993

[2] P. Ganguly, and C.N.R. Rao, Mater. Res. Bull., vol. 8, p.405, 1973

[3] A. C. W. P. James, S. M. Zahurak, and D. W. Murphy, "Superconductivity at $27 \mathrm{~K}$ in fluorine-doped $\mathrm{Nd}_{2} \mathrm{CuO}_{4}$," Nature, vol. 338, pp. 240-241, March 1989

[4] Y. Tokura, H. Takagi, and S. Uchida, "A superconducting oxide compound with electrons as charge carrier," Nature, vol. 337, pp. 345-347, January 1989

[5] J. Gao, W. H. Wong, and J. Xie, "Formation of outgrowths at the initial growing stage of $\mathrm{YBa}_{2} \mathrm{Cu}_{3} \mathrm{O}_{\mathrm{x}}$ ultrathin films on $\mathrm{ZrO}_{2}$ substrates," Appl. Phys. Lett., vol. 67, pp. 2232-2234, October 1995

[6] J. Gao, B. Hăuser, and H. Rogalla, 'High critical current density thin and ultra thin $\mathrm{YBa}_{2} \mathrm{Cu}_{3} \mathrm{O}_{\mathrm{x}}$ films made by a modified rf-magnetron sputtering technique', J. Appl. Phys. Vol. 67, pp. 2512-2515, 1990.

[7] J. Gao, and W. H. Wong, "Investigation on surface morphology of sputtered $\mathrm{YBa}_{2} \mathrm{Cu}_{3} \mathrm{O}_{x}$ thin and ultrathin films," Physica $C$, vol. 251, pp.330336, 1995

[8] Y. Yang, J. Gao, T.C. Chui, and L. Li, "Microstructural sudy of tilted epitaxial thin films of $\mathrm{YBa}_{2} \mathrm{Cu}_{3} \mathrm{O}_{\mathrm{x}}$ with a (105) orientation", Physica $C$, vol. 290, pp. 23-30, 1997

[9] J. G. Wen, C. Traeholt, H. W. Zandbergen, T. Morishita, and N. Koshizuka, "Direct HRTEM observation of non-unit-cell nucleation in the initial stage of high Tc superconducting film growth,"Avances in superconductivity VII, vol. 2, pp. 889-892, November 1994

[10] T. Terashima, K. Shimura, Y. Bando, Y. Matsuda, A. Fujiyama, and S. Komiyama, "Superconductivity of one-unit-cell thick $\mathrm{YBa}_{2} \mathrm{Cu}_{3} \mathrm{O}_{7}$ thin film," Phys. Rev. Lett., vol. 67, pp. 1362-1365, 1991 